\title{
A type I X-ray burst of the Rapid Burster with photospheric radius expansion as seen by Swift
}

\author{
Gloria Sala* \\ Dept. de Física i Enginyeria Nuclear(UPC-IEEC), Comte d'Urgell 187, 08036 Barcelona, Spain \\ E-mail: gloria.saladupc.edu
}

\section{Frank Haberl}

Max-Planck-Institut für extraterrestrische Physik, Giessenbachstr, D-85741, Garching, Germany E-mail: fwhempe.mpg.de

\section{Jordi José}

Dept. de Física i Enginyeria Nuclear (UPC-IEEC) Comte d'Urgell 187, 08036 Barcelona, Spain E-mail: jordi.jose@upc.edu

\section{Anuj Parikh}

Dept. de Física i Enginyeria Nuclear (UPC-IEEC) Comte d'Urgell 187, 08036 Barcelona, Spain E-mail: anuj.parikh@upc.edu

\section{Wolfgang Pietsch}

Max-Planck-Institut für extraterrestrische Physik, Giessenbachstr, D-85741, Garching, Germany E-mail: wnpempe.mpg.de

The Rapid Burster (MXB1730-335) is a unique object showing both type I (thermonuclear) and type II (spasmodic accretion) X-ray bursts. It experiences regular outbursts every 100-200 days, lasting for 2-4 weeks. In January-March 2009 the Rapid Burster experienced a long (20 days) plateau. We obtained 20ks of Swift TOO observations during this period, between February 26 and March 5. A Type I X-ray burst with photospheric radius expansion was observed on 2009 March 5 among type II bursts.

Fast $X$-ray timing and spectroscopy at extreme count rates

February 7-11, 2011

Champéry, Switzerland

\footnotetext{
* Speaker.
} 


\section{Introduction}

The Rapid Burster is a low mass X-ray binary discovered in 1976, located in the Galactic bulge globular cluster Liller 1 (Lewin et al 1976), at a distance of 8-10 kpc from the Earth (Ortolani et al. 2007). A radio transient counterpart was detected (Moore et al 2000), but no IR or optical counterpart has been identified so far (Homer et al. 2001). The Galactic hydrogen column density towards the source is $\mathrm{N}_{H}=(1.2-1.7) \times 10^{22} \mathrm{~cm}^{-2}$ (Dickey \& Lockman 1990; Kalberla et al. 2005). Its behaviour is unique, showing both type I (thermonuclear) and type II (spasmodic accretion) Xray bursts (for a review see Lewin 1995). It is one of the only two sources showing type II bursts, together with the Bursting Pulsar (GRO 1744-28). Type II bursts exhibit no spectral softening and show a range of durations (from $\sim 2 \mathrm{~s}$ to $\sim 700 \mathrm{~s}$ ), with intervals between bursts from $\sim 7 \mathrm{~s}$ to $\sim 1 \mathrm{hr}$. Type I X-ray bursts are due to thermonuclear flashes on the surface of the neutron star and display spectral softening during the decay. They occur at intervals from 1 to 10 hours. At long time-scales, as daily monitored by the RXTE X-ray All Sky Monitor, the Rapid Burster is usually at low intensity ( $<10 \mathrm{mCrab}$ ), showing outbursts (increase to a higher flux) every 100-200 days. Outbursts start with a fast rise up to $100 \mathrm{mCrab}(1.5-12 \mathrm{keV})$, followed by a decay over two to four weeks.

In January-March 2009, the RXTE/ASM showed the Rapid Burster in a long high intensity plateau. We triggered Swift Target of Opportunity observations to follow the type I and type II bursting behaviour during this long outburst. On 2009 March 5, among the type II bursts a bright type I burst with indications of photospheric radius expansion (PRE) was detected.

\section{Observations and data analysis}

Daily monitoring observations were performed between February 26 (MJD 54888.7) and March 5 (MJD 14895.8), accumulating more than $20 \mathrm{ks}$ (Sala et al. 2009). All observations show intense type II bursting activity, with bursts intervals between 25 and 200 seconds. Due to the high average flux of the source, all XRT observations are in Window Timing mode, which minimizes the pile-up at the brightest peaks of the bursts. Most bursts peak at more than $100 \mathrm{cts} / \mathrm{s}$ in the first Swift/XRT (WT) sequences (Feb. 26), which implies fluxes of about $10^{-8} \mathrm{erg} \mathrm{cm}^{2} / \mathrm{s}$ in the range $1-10 \mathrm{keV}\left(\mathrm{L}=(1-1.6) \times 10^{38} \mathrm{erg} / \mathrm{s}\right)$. The peak intensity decreases in the last Swift observations, with most bursts showing maximum intensities of less than $60 \mathrm{cts} / \mathrm{s}$ in XRT (WT) on March 5.

Swift XRT software was used for data reduction (xrtpipeline) and HEASOFT tool xselect for light-curve and spectra extraction. XSPEC was used for spectral analysis. Due to the small count rate of our spectra, C-statistics (Cash 1979) was used for all fits.

Figure 1 shows the Swift/XRT light-curve of the Rapid Burster during the first observation sequence in 2009 March 5 (OBS ID: 00031360008). Six faint type II burst are observed, and one bright burst, with a structured double peak and a long tail is observed among them. The evolution of the hardness ratio (figure [, left panel) indicates clearly the cooling of the photosphere during the decay. For comparison, the light curve and hardness ratio of a type II burst are shown in the right panel of figure 2, showing no cooling during the burst tail.

For time resolved spectral analysis of the cooling tail, spectra have been extracted for small time bins. Time bin duration ranges from 0.9 seconds at the maximum of the burst up to 8.3 

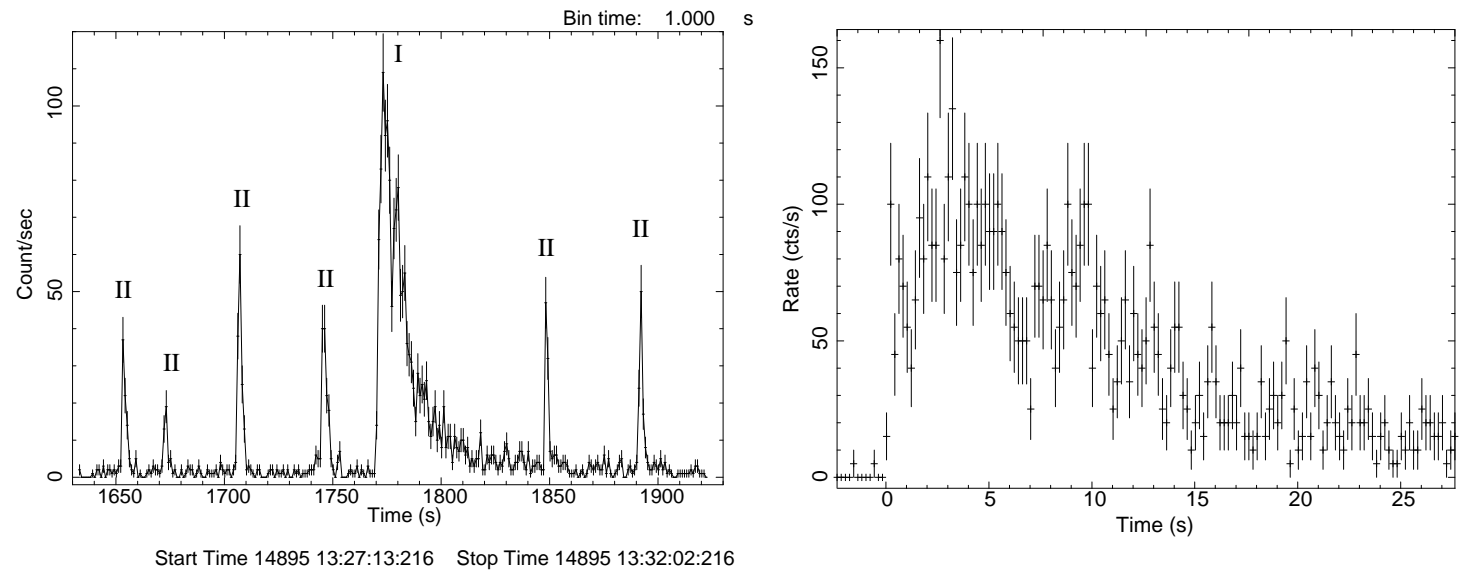

Figure 1: Left panel: $0.2-10 \mathrm{keV}$ lightcurve of the Swift/XRT snapshot in 2009 March 5 containing the type I burst and six type II bursts. Right panel: Swift/XRT light curve $(0.2-10 \mathrm{keV})$ of the Type I X-ray burst.

seconds for the last part of the tail. For each time bin, a spectrum was extracted and fit with an absorbed blackbody model. The Tuebingen-Boulder absorption model (Wilms et al 2000) is used for the interstellar absoprtion. Since statistics in each of the time resolved spectra is low, especially in the soft band, the hydrogen absorbing column was unconstrained in most of our fits. To obtain reliable fits for the blackbody parameters, we fixed the hydrogen density column to its average value towards the source, $1.6 \times 10^{22} \mathrm{~cm}^{-2}$ (Dickey \& Lockman 1990). The evolution of the effective temperature and photospheric radius obtained from the spectral fits is shown in Figure 3 .

\section{Results}

Three clear phases are visible in the PRE Type I X-ray burst light curve and spectral parameters evolution: expansion of the photosphere, during the first rise in count rate, lasting $\sim 3$ seconds; contraction, lasting $\sim 7$ seconds and finishing at the so called touch down of the photosphere, when the photospheric radius falls back down to the neutron star surface; and final cooling down of the envelope.

During expansion, the observed flux and thus the count rate in the X-ray band increase as a consequence of the photospheric radius expansion. A small increase in the rate is observed just at touchdown (secondary peak in the light curve shown in the right panel of figure 1), when the color temperature reaches its maximum and the photospheric radius is almost at its minimum. This may be a geometrical effect caused by the reappearance of the lower hemisphere of the neutron star, hidden by the inner edge of the accretion disk while the photosphere was expanded (see Shaposhnikov, Titarchuk \& Haberl 2003).

The colour (blackbody) temperature decreases during expansion and increases again during the envelope contraction, reaching its maximum at touchdown, when the photosphere reaches the neutron star surface. The cooling of the envelope clearly occurs after the touchdown.

The photospheric radius increases during expansion, and decreases during contraction, reaching its minimum at touchdown, when the photosphere reaches the star surface. A blackbody successfully fits the neutron star observed spectra, but it is well known that the derived temperature 

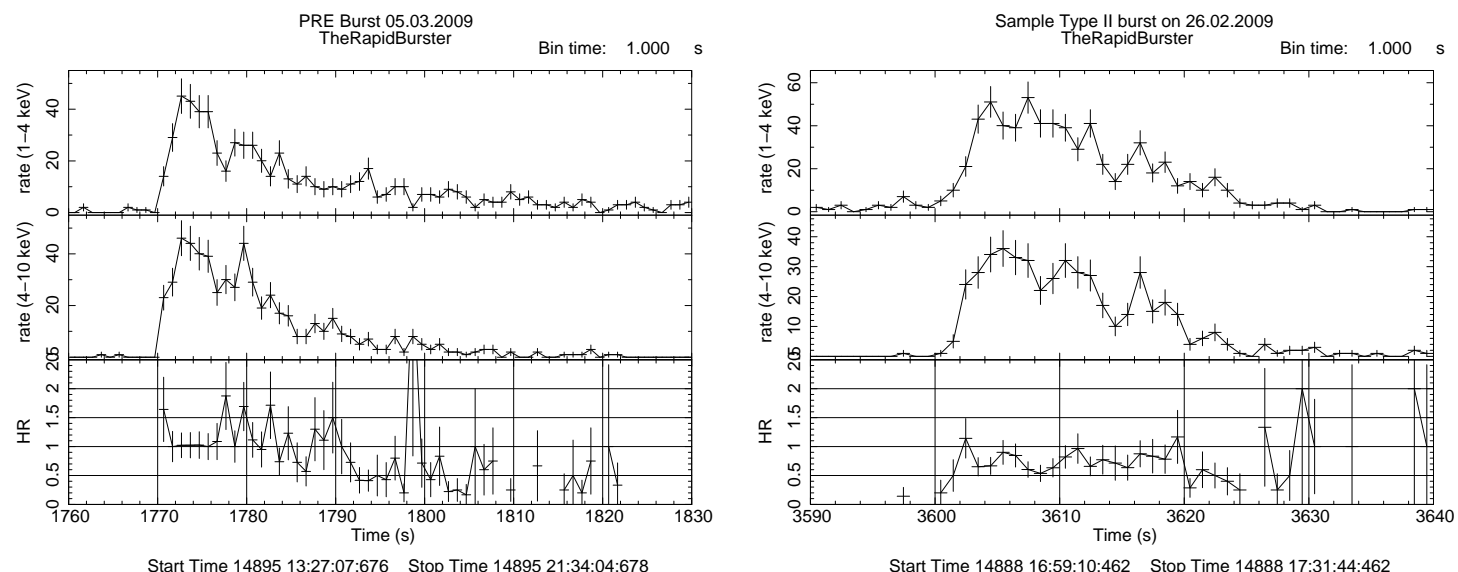

Figure 2: Left panel: Swift/XRT light curve of the Type I X-ray burst on 2009 March 5 in the soft 1-4 keV band (upper panel), the hard 4-10 keV band (middle panel) and the ratio of the two (hard/soft, lower panel). The softening in the burst tail is evident in the ratio. Right panel: Light curves and hardness ratio as in left panel for one of the type II bursts observed by Swift/XRT. No softening is observed in the tail.

is systematically higher than the actual effective temperature due to the hardening of the spectrum in the atmosphere. Assuming a hardening factor $t=T_{b b o d y} / T_{\text {eff }}=1.5$, our observed photospheric radius at touchdown, $\mathrm{R}_{b b o d y}=6.7 \mathrm{~km}$ at $10 \mathrm{kpc}(4.3 \mathrm{~km}$ at $8 \mathrm{kpc})$ would correspond to $\mathrm{R}_{N S}=\mathrm{R}_{b b o d y} t^{2}(1+z)^{-1}=15.1(1+\mathrm{z})^{-1} \mathrm{~km}$ for a distance of $10 \mathrm{kpc}$, or $\mathrm{R}_{N S}=\mathrm{R}_{b b o d y} t^{2}(1+z)^{-1}=$ $9.7(1+\mathrm{z})^{-1} \mathrm{~km}$ for $8 \mathrm{kpc}$.

Expansion of the photosphere in Type I X-ray bursts is caused by the luminosity reaching the Eddington limit. The neutron star mass can be estimated assuming that the touch down (i.e. maximum) luminosity corresponds to the Eddington limit as seen by a distant observer, where $\mathrm{X}$ is the hydrogen abundance and $(1+\mathrm{z})$ is the gravitational redshift at the neutron star surface. For $10 \mathrm{kpc}$ $\left(\mathrm{L}_{\max }=6 \times 10^{38} \mathrm{erg} / \mathrm{s}\right)$ and solar abundances $(\mathrm{X}=0.7)$, the estimated neutron star mass $\mathrm{M}_{N S}$ is 4.1 $(1+\mathrm{z}) \mathrm{M}_{\odot}$, unrealistically large. This may indicate either a smaller distance or higher metalicity. Also for the smallest end of the distance in the accepted range, $8 \mathrm{kpc}$, with solar abundances, the estimated neutron star mass is large, $\mathrm{M}_{N S}=2.5(1+\mathrm{z}) \mathrm{M}_{\odot}$. Since the abundances are actually enriched with respect to the solar values by the nucleosynthesis during the Type I X-ray burst, we adopt more realistic abundances for the mass estimation. José et al (2010) presented a detailed analysis of the nucleosynthesis and nuclear processes involved in thermonuclear X-ray bursts using a hydrodynamic code linked to a nuclear reaction network that contains 325 isotopes and 1392 nuclear processes. Their results show that for a neutron star accreting material with solar abundances, after several Type I X-ray bursts the neutron star envelope has an hydrogen mass fraction of $\mathrm{X}=0.04$ (Burst 4 of Model 1 in José et al 2010). With this we estimate the neutron star mass to be $\mathrm{M}_{N S}=$ $2.5(1+\mathrm{z}) \mathrm{M}_{\odot}$ for $10 \mathrm{kpc}$ and $\mathrm{M}_{N S}=1.6(1+\mathrm{z}) \mathrm{M}_{\odot}$ for $8 \mathrm{kpc}$. This last value is consistent with the average oberved values for neutron star masses in X-ray binaries (Lattimer \& Prakash 2006) and favours both the higher metalicity in the envelope and the smaller distance, $8 \mathrm{kpc}$. 

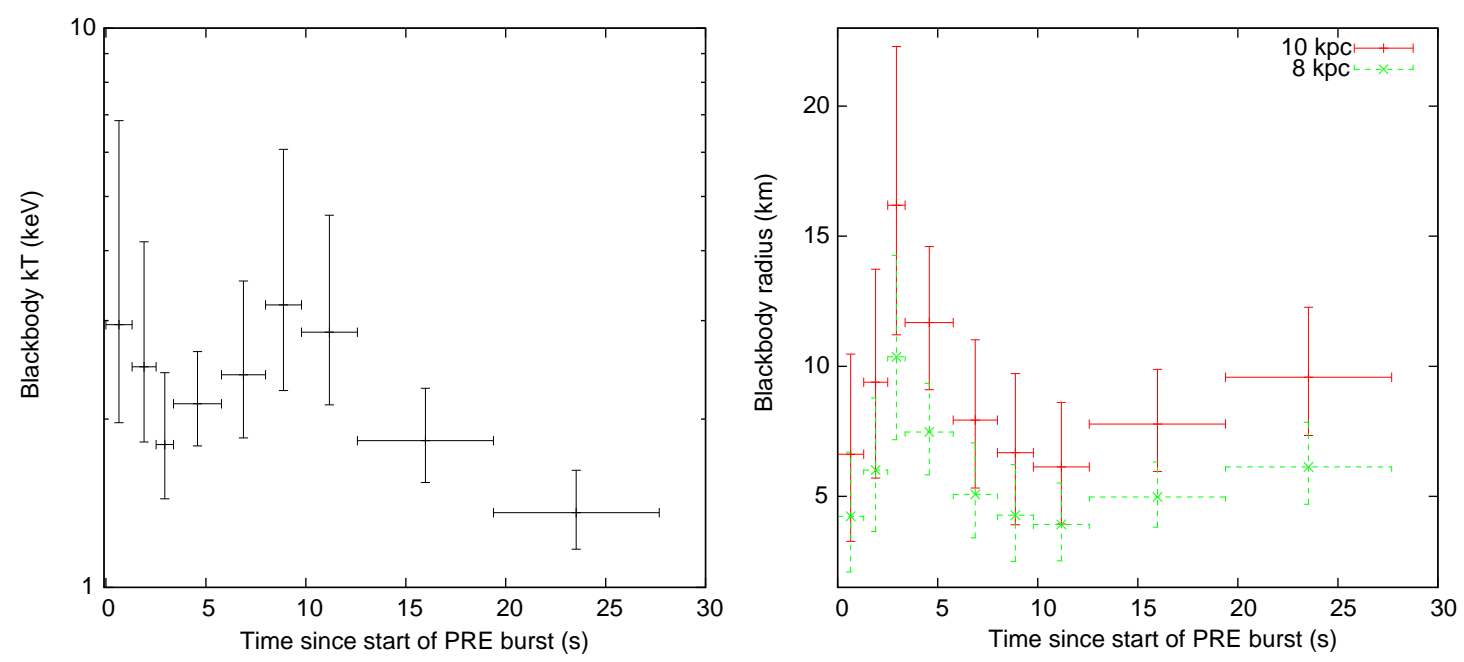

Figure 3: Time resolved spectral analysis: evolution of the spectral parameters during the type I X-ray burst.

\section{Acknowledgments}

We thank the Swift team and its PI Niel Gehrels for scheduling these TOO observations. This research has been funded by the MICINN grants AYA2008-04211-C02-01 and AYA2010-15685, and funds from ESF EUROCORES Program EuroGENESIS through grant EUI2009-04167

\section{References}

[1] K. Arnaud, B. Dorman, C. Gordon 2010, XSPEC v12.6.0 (NASA/GSFC, MD)

[2] W. Cash 1979, ApJ, 228, 939

[3] J.M. Dickey \& F. J. Lockman 1990, ARAA, 28, 215

[4] P.M.W. Kalberla, W.B. Burton, D. Hartmann et al. 2005, A\&A, 440, 775

[5] L. Homer, E.W. Deutsch, S.F. Anderson, B. Margon 2001 ApJ, 122, 2627

[6] J. José, F. Moreno, A. Parikh, C. Iliadis 2010 ApJS 189, 204

[7] J.M. Lattimer \& M. Prakash 2006, NucPhys A 777, 479

[8] A. Levine \& R. Remillard 2009, ATel \#1939

[9] W. Lewin et al. 1976, ApJ, 207, L95

[10] W. Lewin, J. van Paradijs, R. Taam in X-ray binaries, edited by Lewin, van Paradijs \& van den Heuvel, Camb. Astrophys. Ser., Vol. 26, 1995, pp. 175-232

[11] C. B. Moore, R. E. Rutledge, D. W. Fox et al. 2000, ApJ 532, 1181

[12] S. Ortolani, B. Barbuy, E. Bica, M. Zoccali, A. Renzini 2007 A\&A, 470, 1043

[13] G. Sala, F. Haberl, W. Pietsch, J. José, A. Parikh 2009, ATel \#1969

[14] N. Shaposhnikov, L. Titarchuk, F. Haberl 2003, ApJ, 593, L35

[15] J. Wilms, A. Allen, R. McCray 2000, ApJ, 542, 914

[16] M. V. Zombeck 2007, Handbook of Space Astronomy and Astrophysics (Cambridge Univ. Press, 2007,3 rd ed) 